\title{
Tidal tails in the disintegrating open cluster NGC 752
}

\author{
Souradeep Bhattacharya, ${ }^{1 \star}$ Manan Agarwal, ${ }^{2}$ Khushboo K. Rao ${ }^{2}$ and Kaushar Vaidya ${ }^{2}$ \\ ${ }^{1}$ Inter University Centre for Astronomy and Astrophysics, Ganeshkhind, Post Bag 4, Pune 411007, India \\ ${ }^{2}$ Department of Physics, Birla Institute of Technology and Science - Pilani, Rajasthan 333031, India
}

Accepted 2021 May 12. Received 2021 May 10; in original form 2021 April 12

\begin{abstract}
We utilize the robust membership determination algorithm, ML-MOC, on the precise astrometric and deep photometric data from Gaia Early Data Release 3 within a region of radius $5^{\circ}$ around the center of the intermediate-age galactic open cluster NGC 752 to identify its member stars. We report the discovery of the tidal tails of NGC 752, extending out to $\sim 35$ pc on either side of its denser central region and following the cluster orbit. From comparison with PARSEC stellar isochrones, we obtain the mass function of the cluster with a slope, $\chi=-1.26 \pm 0.07$. The high negative value of $\chi$ is indicative of a disintegrating cluster undergoing mass-segregation. $\chi$ is more negative in the intra-tidal regions as compared to the outskirts of NGC 752. We estimate a present day mass of the cluster, $\mathrm{M}_{\mathrm{C}}=297 \pm 10 \mathrm{M}_{\odot}$. Through mass-loss due to stellar evolution and tidal interactions, we further estimate that NGC 752 has lost nearly $95.2-98.5 \%$ of its initial mass, $\mathrm{M}_{\mathrm{i}}=0.64-2 \times 10^{4} \mathrm{M}_{\odot}$.
\end{abstract}

Key words: methods: data analysis - open clusters and associations: individual : NGC 752

\section{INTRODUCTION}

Almost all stars ever formed were likely born in clustered environments (see review by Portegies Zwart et al. 2010, and references therein). Stars inside a cluster are subjected to two-body relaxation which over time leads to more-massive stars sinking to the central regions of the cluster while less-massive stars occupy a larger volume, many of which gradually evaporate to supply the field population of a galaxy. This effect is called mass segregation and has been observed in both galactic globular (von Hoerner 1957) and open (Mathieu 1984) clusters, investigated both through the slope of the mass-function of the cluster members in the core and outskirts (e.g. Bhattacharya et al. 2017b), as well as through the radial distribution of massive objects, such as blue stragglers (e.g. Bhattacharya et al. 2019; Vaidya et al. 2020; Rao et al. 2021). Alongside this internal effect, Galactic potential perturbs star clusters leading to the formation of tidal structures. Such tidal structures are observed in both globular clusters (e.g. Palomar 5 - Odenkirchen et al. 2001; NGC 5466 - Belokurov et al. 2006) and open clusters (e.g. Berkeley 17 - Chen et al. 2004; Bhattacharya et al. 2017b; NGC 6791 - Dalessandro et al. 2015).

Galactic open clusters, in particular, are more abundant in the disc where they are additionally subjected to external gravitational effects such as interactions with giant molecular clouds and passage through spiral arms (Spitzer 1958). From an observational standpoint, the identification of open cluster morphology, despite the use of novel techniques (e.g. Bhattacharya et al. 2017a), has long since relied on stellar density determination against strong field contamination in photometric observations. Low-density features such as tidal tails have thus been particularly difficult to identify with little success (Chen et al. 2004; Dalessandro et al. 2015). Reliable astrometry

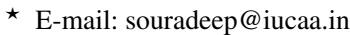

(proper motion and parallax) from Gaia Data Release 2 (Gaia DR2; Gaia Collaboration et al. 2018) and recently Gaia Early Data Release 3 (Gaia EDR3; Gaia Collaboration et al. 2020) has allowed for improved field-star decontamination of many nearby clusters through novel membership determination techniques (Agarwal et al. 2021). This has resulted in the recent discovery of a few open clusters with extended stellar coronae (e.g. Carrera et al. 2019; Meingast et al. 2021) as well as those with tidal features - Hyades: Röser et al. (2019); Coma Berenices: Tang et al. (2019); Ruprecht 147: Yeh et al. (2019); Praesepe: Röser \& Schilbach (2019); NGC 2506: Gao (2020b); M 67: Gao (2020a); UBC 274: Castro-Ginard et al. (2020); Alpha Persei: Nikiforova et al. (2020).

NGC 752 (cluster center at RA=29.156 deg and DEC=37.809 deg; Agarwal et al. 2021) is an intermediate age ( 1.5 Gyr old; Agüeros et al. 2018), nearby ( 450 pc) open cluster which was among the clusters whose membership determination was carried out by Agarwal et al. (2021) using Gaia DR2 data with their novel membership determination algorithm, ML-MOC. Within their search radius of $2.5^{\circ}$ around its center, Agarwal et al. (2021) found hints of tidal tails in NGC 752. Hu et al. (2021) also identify the elongated morphology of the peripheral regions of NGC 752 from the Gaia DR2 data using the members identified by Cantat-Gaudin et al. (2018).

In this paper, we report the discovery of the tidal tails of NGC 752 within a large spatial region around the cluster. The data and membership determination are presented in Section 2. The analysis of the mass function and tidal structure of NGC 752 is presented in Section 3. Finally, we discuss the orbit of the cluster and its dissolution in Section 4.

\section{DATA AND MEMBERSHIP SELECTION}

Gaia EDR3 provides the positions, trigonometric parallaxes $(\omega)$, and proper motions (PM) as well as photometry in three broad- 

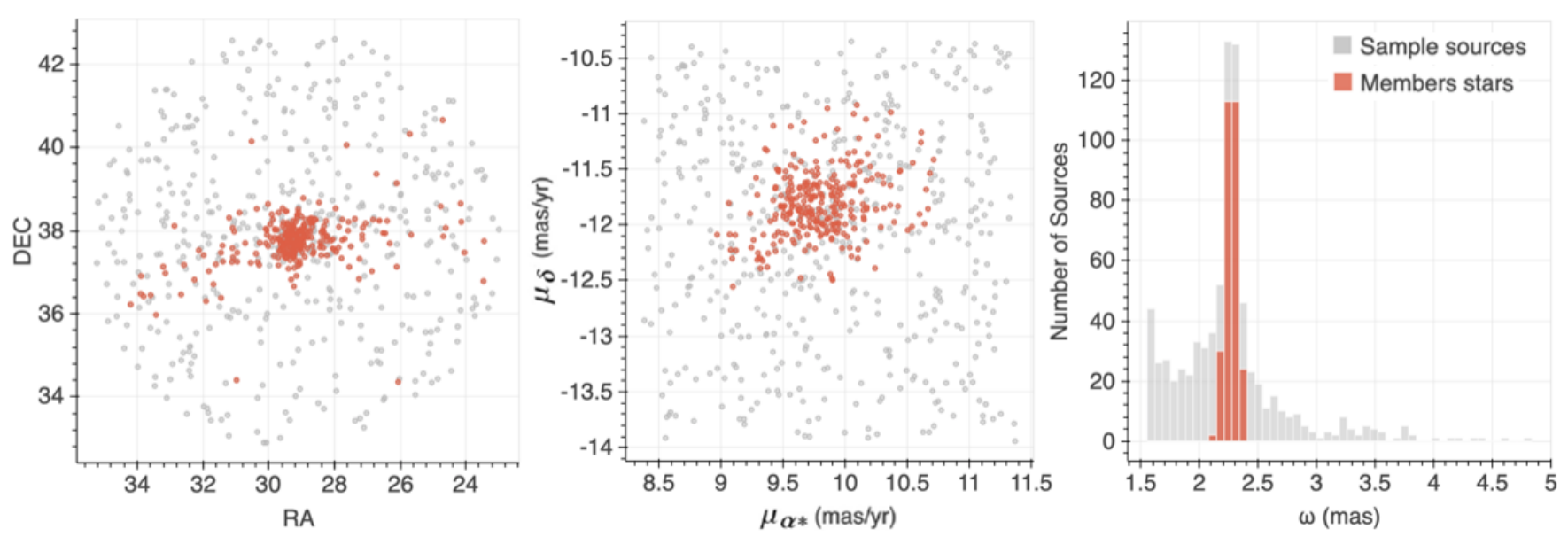

Figure 1. The spatial, proper-motion and parallax distributions of the Sample sources and the member stars identified by ML-MOC.

Table 1. Gaia EDR3 members identified in NGC 752 using ML-MOC. The full table will be available in the online version of the journal.

\begin{tabular}{|c|c|c|c|c|c|c|c|c|c|c|}
\hline GaiaID & $\begin{array}{l}\text { RA } \\
\text { deg }\end{array}$ & $\begin{array}{c}\text { DEC } \\
\text { deg }\end{array}$ & $\begin{array}{c}\omega \\
\text { mas }\end{array}$ & $\begin{array}{c}\mu_{\alpha *} \\
\mathrm{mas} / \mathrm{yr}\end{array}$ & $\begin{array}{c}\mu_{\delta} \\
\mathrm{mas} / \mathrm{yr}\end{array}$ & $\underset{\mathrm{mag}}{\mathrm{G}}$ & $\begin{array}{c}\text { BP } \\
\text { mag }\end{array}$ & $\begin{array}{l}\text { RP } \\
\text { mag }\end{array}$ & $\begin{array}{c}\mathrm{RV} \\
\mathrm{km} / \mathrm{s}\end{array}$ & $\mathrm{p}_{\mathrm{memb}}$ \\
\hline 342914075859711744 & 29.23240573 & 37.79978973 & 2.288 & 9.803 & -11.878 & 11.1 & 11.3 & 10.75 & 6.91 & 0.98 \\
\hline 342912284857245952 & 29.15366539 & 37.75349579 & 2.272 & 9.892 & -12.043 & 13.64 & 14.04 & 13.07 & - & 0.97 \\
\hline 342914556896034176 & 29.22108601 & 37.8692465 & 2.261 & 9.462 & -11.507 & 10.15 & 10.38 & 9.77 & 18.0 & 0.92 \\
\hline 342915411593428736 & 29.16345994 & 37.86139137 & 2.267 & 9.721 & -11.886 & 10.29 & 10.48 & 9.96 & 8.23 & 0.98 \\
\hline 342912254793586688 & 29.13259369 & 37.74945795 & 2.141 & 9.794 & -11.62 & 16.2 & 17.08 & 15.27 & - & 0.68 \\
\hline 342913178210782080 & 29.11199587 & 37.79182891 & 2.231 & 9.854 & -12.06 & 17.83 & 19.17 & 16.72 & - & 0.95 \\
\hline 342909845316110336 & 29.31580848 & 37.73702508 & 2.363 & 9.887 & -11.726 & 18.3 & 19.57 & 17.16 & - & 0.91 \\
\hline 342920501130956800 & 29.26040912 & 37.88539331 & 2.235 & 9.815 & -11.984 & 11.21 & 11.43 & 10.84 & 5.1 & 0.97 \\
\hline 342920501130956928 & 29.26070082 & 37.88616266 & 2.219 & 10.006 & -11.428 & 13.9 & 14.2 & 13.24 & - & 0.92 \\
\hline 342921257045010432 & 29.22252061 & 37.8972341 & 2.169 & 9.499 & -11.925 & 14.92 & 15.52 & 14.18 & - & 0.9 \\
\hline
\end{tabular}

band filters (G, BP, and RP) for almost 1.5 billion stars. It improves upon the Gaia DR2 with the proper motion uncertainty reduced on average by nearly a factor of two while parallax measurements are more accurate by $\sim 20 \%$ (Gaia Collaboration et al. 2020). This allows for reliable photometry down to $\mathrm{G} \sim 21 \mathrm{mag}$. Gaia EDR3 photometry is essentially complete down to $\mathrm{G}=17$ mag although the completeness may be lower in regions of high stellar density such as those of globular clusters (Fabricius et al. 2020). Radial velocity measurements in Gaia EDR3 are nearly the same as in Gaia DR2. Further details on Gaia EDR3 are available at https://www. cosmos.esa.int/web/gaia/earlydr3.

The membership selection in NGC 752 from the Gaia EDR3 dataset is carried out in a region of radius $5^{\circ}$ around its center. This sample is termed All sources. We use ML-MOC (Agarwal et al. 2021) to identify cluster members using the proper motion and parallax information. While details of the membership selection are described in Agarwal et al. (2021), we briefly state it here. ML-MOC is based on the k-nearest neighbour algorithm (kNN; Cover \& Hart 1967) and the Gaussian mixture model (GMM; Mclachlan \& Peel 2000). It identifies cluster members in the PM- $\omega$ parameter space, independent of the spatial density of the cluster, thereby allowing for the identification of faint extended spatial structures such as tidal tails. As a first step to the membership selection, $\mathrm{kNN}$ is utilised to remove the obvious field stars such that the remaining member candidates have more cluster members than field stars. This is done by applying $\mathrm{kNN}$ to those stars within the central region of radius $1^{\circ}$ to calculate a broad range of PM and $\omega$ values for the cluster members. For NGC 752, we find the range of PM in RA, $\mu_{\alpha *}=8.372-11.372$ mas/yr, PM in DEC, $\mu_{\delta *}=-13.948--10.348$ mas/yr, and $\omega=1.552$ - 5.199. The stars in All sources which fall in the aforementioned parameter ranges are termed Sample sources. Their spatial, PM and $\omega$ distributions are shown in Figure 1. For the second step, a three dimensional GMM is used in the PM- $\omega$ parameter space of the Sample sources to distinguish between the cluster and field members, also assigning a membership probability $\left(\mathrm{p}_{\mathrm{memb}}\right)$. Those sources having $\mathrm{p}_{\text {memb }} \geq 0.6$ are considered as high probability members, also including all the radial velocity members. A small number of stars having $0.2 \leq \mathrm{p}_{\text {memb }} \leq 0.6$ are also considered as members if their $\omega$ values lie within the range of $\omega$ values specified by the members that have $\mathrm{p}_{\text {memb }} \geq 0.8$. This results in the identification of 282 members in NGC 752 whose spatial, PM and $\omega$ distributions are also shown in Figure 1. Note the extended tails are clearly identified in the spatial distribution. The identified members with their Gaia EDR3 IDs, astrometry, photometry, radial velocity (RV) and $\mathrm{p}_{\text {memb }}$ have been tabulated in Table 1.

In Figure $2 \mathrm{a}$, the marked $\mathrm{p}_{\text {memb }}$ values show that the extended tails are present for the high probability members showing their identification as being robust. The mean distance to the cluster is found to be 443.8 pc (see Agarwal et al. 2021) consistent with literature values (Agüeros et al. 2018). The cluster members with RV data from Gaia EDR3 are shown in Figure 2b, having $<R V>=7.88 \mathrm{~km} / \mathrm{s}$ and $\sigma_{\mathrm{RV}}=5.03 \mathrm{~km} / \mathrm{s}$. Few cluster members with RV data are also identi- 

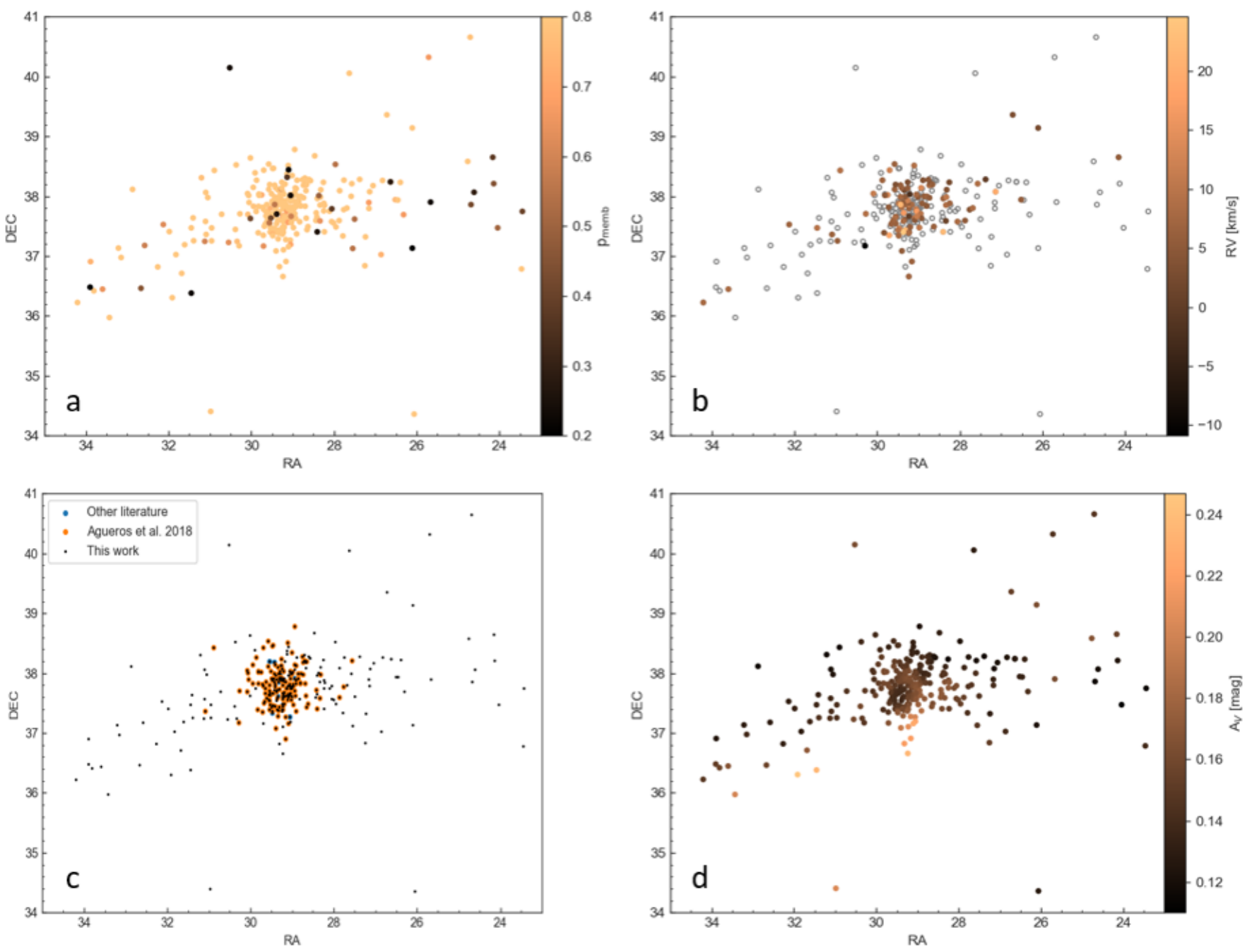

Figure 2. Spatial distribution of the identified members in NGC 752. (a) Coloured by their pmemb values. (b) Coloured by their RV values where available and open grey circles where unavailable. (c) Matched previously-identified members. (d) Coloured by their AV values.

fied among the extended features. Agüeros et al. (2018) had identified 258 members in NGC 752 from photometry and PM from the TychoGaia Astrometric Solution (TGAS) catalog (Gaia Collaboration et al. 2016). Furthermore, they had consolidated membership determinations from previous studies (primarily Daniel et al. 1994; Mermilliod et al. 1998) finding 32 literature members as non-members. 144 of the stars identified by (Agüeros et al. 2018) as members have counterparts within $1^{\prime \prime}$ of our identified members while 14 of the literature members classified as non-members also have counterparts within $1^{\prime \prime}$ of our identified members. The culling of non-members in our membership sample as compared to those in the literature is testament to the improved accuracy of the Gaia EDR3 astrometry and our robust membership determination from ML-MOC. Figure $2 \mathrm{c}$ shows that the literature members are concentrated in the central parts of NGC 752 as the extended tails were not surveyed in previous spatially-restrictive studies whose membership determinations were guided by cluster radial density.

\section{ANALYSIS}

\subsection{Reddening}

We utilise the interstellar reddening, $E(B-V)$, map provided by Schlafly \& Finkbeiner (2011) available through the NASA/IPAC Infrared Science Archive ${ }^{1}$ to obtain the line-of-sight extinction $\left(A_{V}=3.1 \times E(B-V)\right.$; Cardelli et al. 1989) towards each identified cluster member in NGC 752 . Figure $2 d$ shows the spatial distribution of the cluster members coloured by their $A_{V}$. The mean extinction $<A_{V}>=0.15 \mathrm{mag}$. The extinction for each cluster member is converted to the Gaia photometric system following the extinction law by Wang \& Chen (2019). There is indeed some evidence of differential reddening but only a small range of extinction values is covered.

\subsection{Color Magnitude Diagram and Mass Function}

Correcting for extinction and reddening for each individual cluster member, we obtain the de-reddened color magnitude diagram (CMD)

1 https://irsa.ipac.caltech.edu/applications/DUST 

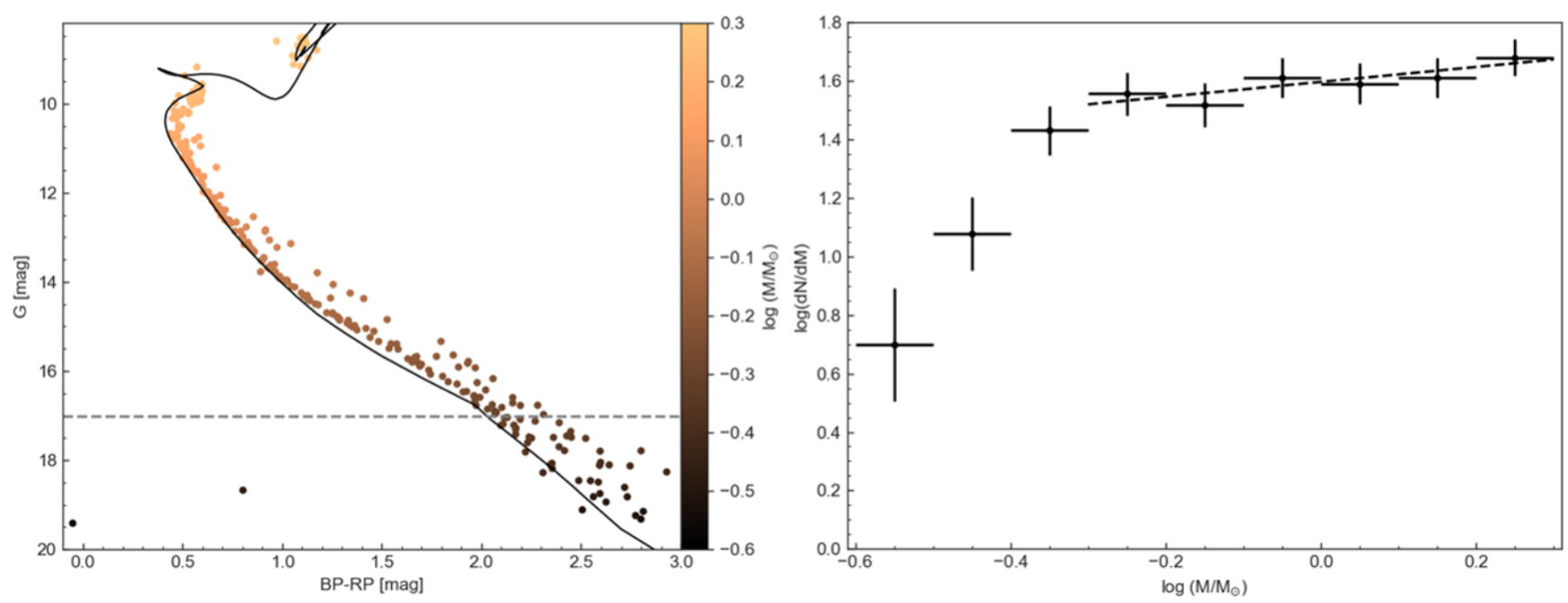

Figure 3. [Left] CMD of all the identified members in NGC 752, coloured by their stellar masses estimated from the PARSEC isochrone (black). The dashed grey line shows the $\sim 100 \%$ completeness limit of the Gaia EDR3 data. [Right] The Mass function derived from the PARSEC iscochrones for the entire cluster. The dashed black line shows the fitted mass function where the Gaia EDR3 data is almost complete. The Poissonian uncertainties have been marked.

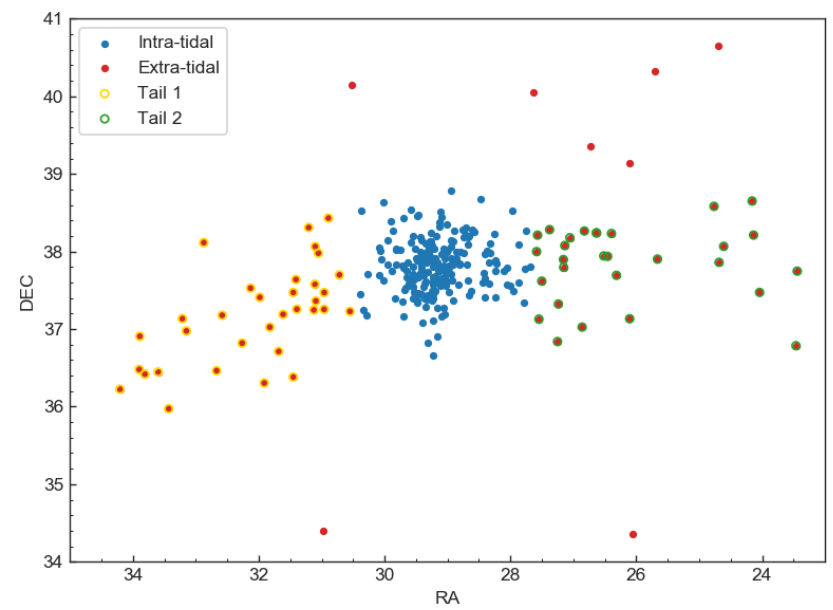

Figure 4. Spatial distribution of the identified members in NGC 752 with the intra-tidal (blue), extra-tidal (red), Tail 1 (encircled in yellow) and Tail 2 (encircled in green) members marked.

of the identified members shown in the left panel of Figure 3. The PARSEC stellar evolution isochrone ${ }^{2}$ (Bressan et al. 2012; Chen et al. 2014) for the Gaia filters has been plotted for the obtained distance corresponding to an age, $\mathrm{t}=1.5 \mathrm{Gyr}$ and $[\mathrm{M} / \mathrm{H}]=-0.04$, in line with fitted literature values of age $(\mathrm{t}=1.45 \pm 0.05 \mathrm{Gyr})$ from multi-wavelength photometry by Twarog et al. (2015) and metallicity $(<[\mathrm{M} / \mathrm{H}]>=-0.07 \pm 0.04)$ from spectroscopy of the red giant stars by Böcek Topcu et al. (2015).

Stellar mass of each star was estimated from the PARSEC isochrone and is shown in the left panel of Figure 3. The total observed mass of the cluster members is $284 \mathrm{M}_{\odot}$. The mass function of all the identified members in NGC 752 is shown in the right panel of Figure 3. The mass function is fitted above the stellar mass value

2 http://stev.oapd.inaf.it/cgi-bin/cmd $\left(\approx 0.5 \mathrm{M}_{\odot}\right)$ corresponding to the Gaia EDR3 completeness limit of $\mathrm{G}=17$ mag. Using the relation $\log (\mathrm{dN} / \mathrm{dM})=-(1+\chi) \times \log (\mathrm{M})+$ constant, where $\mathrm{dN}$ represents the number of stars in a mass bin $\mathrm{dM}$ with central mass $\mathrm{M}$, we obtain the slope of the mass function for NGC 752, $\chi=-1.26 \pm 0.07$. Such a negative value of $\chi$, as compared to its value of $\chi=1.37$ as derived by (Salpeter 1955) for the solar neighbourhood, is indicative of a dissolving cluster that has undergone significant mass segregation. Extrapolating the fitted mass function to the stellar hydrogen burning limit of $0.08 \mathrm{M}_{\odot}$, we obtain the total mass of the cluster, $\mathrm{M}_{\mathrm{C}}=297 \pm 10 \mathrm{M}_{\odot}$.

\subsection{Tidal Structure}

While extended tails are clearly visible in the spatial distribution of NGC 752 (Figure 2), we need to estimate the tidal radius $\left(r_{t}\right)$ of the cluster in order to ascertain the extra-tidal nature of these tails. As estimating the tidal radius from fitting the King function (King 1966) to the radial density of the cluster (as carried out by Agarwal et al. 2021) is not appropriate for a dissolving cluster with extended structures, we calculate $r_{t}$ following Pinfield et al. (1998) as:

$\mathrm{r}_{\mathrm{t}}^{3}=\frac{\mathrm{GM}_{\mathrm{C}}}{2(\mathrm{~A}-\mathrm{B})^{2}}$

where $\mathrm{G}$ is the gravitational constant, and $\mathrm{A}$ and $\mathrm{B}$ are the Oort constants $(\mathrm{A}=15.3 \pm 0.4 \mathrm{~km} / \mathrm{s} / \mathrm{kpc}, \mathrm{B}=-11.9 \pm 0.4 \mathrm{~km} / \mathrm{s} / \mathrm{kpc}$; Bovy 2017). We calculate $r_{t}=1.2123^{\circ}=9.52 \mathrm{pc}$ at the distance of NGC 752. The identified members within and beyond $r_{t}$ are classified as intra-tidal and extra-tidal members respectively (Figure 4). Amongst the extra-tidal members, we further note those stars belonging to either of the two tails $(1 \& 2$; see Figure 4$)$. At the distance of NGC 752, tail 1 and 2 extend out to $\sim 34$ and $\sim 36.5$ pc respectively from the center of the cluster.

We further obtain the de-reddened CMDs for the four sub-regions of NGC 752 (intra-tidal, extra-tidal, Tail 1 and Tail 2) as shown in the left panel of Figure 5. The mass functions for each of the sub-regions are obtained and fitted as shown in the right panel of Figure 5. The fitted slopes of the mass functions are $\chi=-1.44 \pm 0.12,-0.69 \pm 0.49$, $-0.87 \pm 0.57$ and $-0.63 \pm 0.64$ for the intra-tidal, extra-tidal, Tail 1 and Tail 2 respectively. The mass function slope is most negative in 

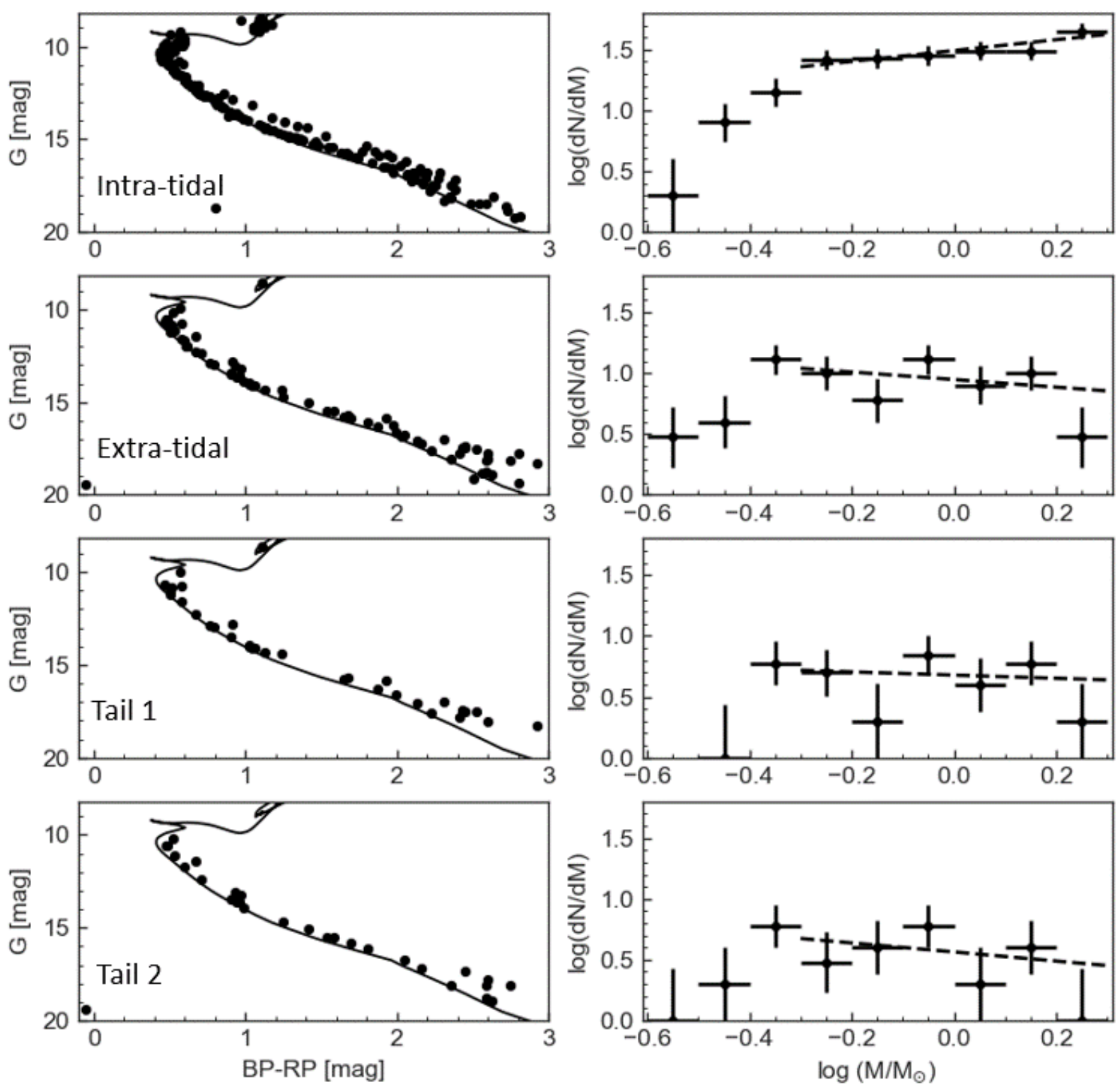

Figure 5. [Left] De-reddened CMD of all the identified members in NGC 752 in the four different regions. The black line shows the PARSEC isochrone corresponding to the age and metallicity of NGC 752. [Right] The Mass function derived from the PARSEC iscochrones for each region. The dashed black line shows the fitted mass function where the Gaia EDR3 data is almost complete. The Poissonian uncertainties have been marked.

the intra-tidal region, as expected in case of mass segregation, while it is relatively less-steep outside $r_{t}$ despite the large uncertainty.

\section{DISCUSSION}

\subsection{Cluster orbit}

To further validate the tidal origin of the extended tails, we explore their position with respect to the cluster orbit in a galactocentric coordinate system, similar to that carried out by Tang et al. (2019) for Coma Berenices. The Galactocentric coordinates have a positive $\mathrm{x}$ direction pointing from the position of the Sun projected to the Galactic midplane to the Galactic center (GC); the y-axis points toward $l=90^{\circ}$ and the z-axis roughly points toward $b=90^{\circ}$. Distance to the GC is $8.3 \mathrm{kpc}$ (Reid \& Brunthaler 2004). Figure 6 shows the
$\mathrm{X}-\mathrm{Y}$ (left), Y-Z (middle) and X-Z (right) projections of the cluster members.

We compute the average position of all member stars as the cluster mean position giving $X=-8595.62 \mathrm{pc}, \mathrm{Y}=276.26 \mathrm{pc}, \mathrm{Z}=-152.36$ $\mathrm{pc}$, and its mean space velocity (for only those sources having RV measurements) as $U=-5.13 \mathrm{~km} / \mathrm{s}, \mathrm{V}=226.44 \mathrm{~km} / \mathrm{s}, \mathrm{W}=-12.03 \mathrm{~km} / \mathrm{s}$. The aforementioned are utilised to calculate the cluster orbit using the python package galpy (Bovy 2015) which accounts for solar motion and includes the Milky Way potential model MWPotential2014 (A fitted Galactic potential model incorporating the bulge, disc and halo). Orbits are integrated $100 \mathrm{Myr}$ backward and $100 \mathrm{Myr}$ forward in time.

Figure 6 shows the orbit motion of NGC 752 in the three galactocentric projections. The distribution of the extended tails along the cluster orbit is most prominent in the Y-Z plane, similar to that found by Röser et al. (2019), Tang et al. (2019) and Yeh et al. (2019) for 

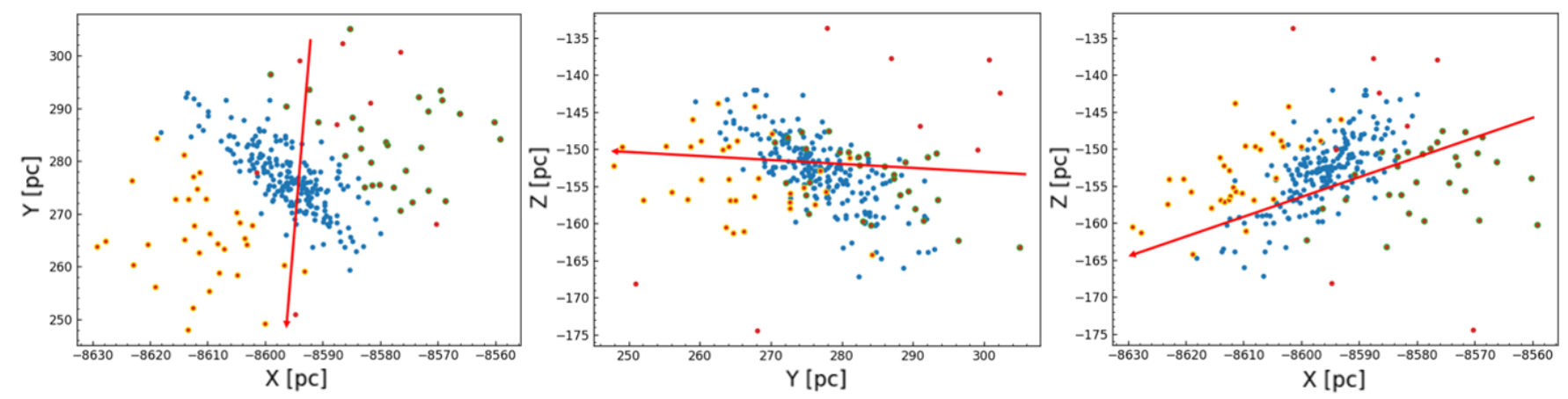

Figure 6. Tri-dimensional projections in the galactocentric coordinate system of the identified members in NGC 752 with the intra-tidal (blue), extra-tidal (red), Tail 1 (encircled in yellow) and Tail 2 (encircled in green) members marked. The red arrow follows the cluster orbit.

their studied clusters. This is a testament to the tidal nature of the extended tails of NGC 752 with Tails $1 \& 2$ being the leading and trailing tails respectively. Through reliable membership determination up to large radii in conjunction with reliable astrometry from Gaia EDR3, NGC 752 is now added to a growing list of dissolving open clusters exhibiting tidal features.

\subsection{Initial cluster mass}

The mass-segregation of NGC 752, evident from the negative value of its mass function slope, and extended tidal features mark NGC 752 as a telltale sign of its dissolution. Environmental effects like tidal shocks due to close interactions with giant molecular clouds, spiral arms, the Galactic disc and, in general, interactions with the Galactic tidal field or internal dynamical effects like two-body relaxation lead to mass-loss in an open cluster (Dalessandro et al. 2015).

Lamers et al. (2005) provide an analytical prescription to estimate the mass lost by an open cluster due to the Galactic tidal field. The initial mass $\left(\mathrm{M}_{\mathrm{i}}\right)$ is estimated with the following relation:

$\mathbf{M}_{i}=\left[\left(\frac{\mathrm{M}_{\mathrm{C}}}{\mathrm{M}_{\odot}}\right)^{\gamma}+\frac{\gamma \mathrm{t}}{\mathrm{t}_{0}}\right]^{\frac{1}{\gamma}}\left[1-\mathrm{q}_{\mathrm{ev}}(\mathrm{t})\right]^{-1}$

where $t_{0}$ is the dissolution time-scale parameter. By comparing the distribution of mass and age of OCs in the solar neighbourhood with theoretical predictions, Lamers et al. (2005) obtained $t_{0}=3.3_{-1.0}^{1.4}$ Myr. $\gamma$ is a dimensionless index which depends on the cluster initial density distribution. We adopt $\gamma=0.62$ which is the typical value for open clusters (Lamers et al. 2005; Dalessandro et al. 2015). The function $\mathrm{q}_{\mathrm{ev}}(\mathrm{t})$ describes the mass-loss due to stellar evolution and can be approximated by the following analytical formula:

$\mathrm{q}_{\mathrm{ev}}(\mathrm{t})=\left(\log _{10}(\mathrm{t})-\mathrm{a}\right)^{\mathrm{b}}+\mathrm{c}$

where $\mathrm{a}=7.03, \mathrm{~b}=0.26$ and $\mathrm{c}=-1.80$ for the metallicity of NGC 752 (see Table 1 in Lamers et al. 2005). Plugging the values in to Equations $2 \& 3$, we find $\mathrm{M}_{\mathrm{i}}=11087_{-4666}^{8922}$, i.e, $\mathrm{M}_{\mathrm{i}}=0.64-$ $2 \times 10^{4} \mathrm{M}_{\odot}$. Thus, NGC 752 is a descendent of a Young Massive Cluster (which have stellar masses $\gtrsim 10^{4} \mathrm{M}_{\odot}$ ) such as those observed in the Milky Way and other galaxies (see review by Portegies Zwart et al. 2010) that has lost 95.2-98.5\% of its mass to the Galactic field.

\section{ACKNOWLEDGEMENTS}

This work presents results from the European Space Agency (ESA) space mission Gaia. Gaia data are being processed by the Gaia Data
Processing and Analysis Consortium (DPAC). Funding for the DPAC is provided by national institutions, in particular the institutions participating in the Gaia MultiLateral Agreement (MLA). We thank the anonymous referee for their comments. This research made use of Astropy- a community-developed core Python package for Astronomy (Astropy Collaboration et al. 2013), SciPy (Virtanen et al. 2020), NumPy (Harris et al. 2020) and Matplotlib (Hunter 2007). This research also made use of NASA's Astrophysics Data System $\left(\mathrm{ADS}^{3}\right)$.

\section{DATA AVAILABILITY}

The data underlying this article are publicly available at https: //archives.esac.esa.int/gaia. The 282 identified members of NGC 752 along with their Gaia EDR3 identifiers and photometric and astrometric data have been presented in Table 1.

\section{REFERENCES}

Agarwal M., Rao K. K., Vaidya K., Bhattacharya S., 2021, MNRAS, 502, 2582

Agüeros M. A., et al., 2018, ApJ, 862, 33

Astropy Collaboration et al., 2013, A\&A, 558, A33

Belokurov V., Evans N. W., Irwin M. J., Hewett P. C., Wilkinson M. I., 2006, ApJ, 637, L29

Bhattacharya S., Mahulkar V., Pandaokar S., Singh P. K., 2017a, Astronomy and Computing, 18,

Bhattacharya S., Mishra I., Vaidya K., Chen W. P., 2017b, ApJ, 847, 138

Bhattacharya S., Vaidya K., Chen W. P., Beccari G., 2019, A\&A, 624, A26

Böcek Topcu G., Afşar M., Schaeuble M., Sneden C., 2015, MNRAS, 446, 3562

Bovy J., 2015, ApJS, 216, 29

Bovy J., 2017, MNRAS, 468, L63

Bressan A., Marigo P., Girardi L., Salasnich B., Dal Cero C., Rubele S., Nanni A., 2012, MNRAS, 427, 127

Cantat-Gaudin T., et al., 2018, A\&A, 618, A93

Cardelli J. A., Clayton G. C., Mathis J. S., 1989, ApJ, 345, 245

Carrera R., et al., 2019, A\&A, 627, A119

Castro-Ginard A., et al., 2020, A\&A, 635, A45

Chen W. P., Chen C. W., Shu C. G., 2004, AJ, 128, 2306

Chen Y., Girardi L., Bressan A., Marigo P., Barbieri M., Kong X., 2014, MNRAS, 444, 2525

Cover T., Hart P., 1967, IEEE Transactions on Information Theory, 13, 21

3 https://ui.adsabs.harvard.edu 
Dalessandro E., Miocchi P., Carraro G., Jílková L., Moitinho A., 2015, MNRAS, 449, 1811

Daniel S. A., Latham D. W., Mathieu R. D., Twarog B. A., 1994, PASP, 106, 281

Fabricius C., et al., 2020, arXiv e-prints, p. arXiv:2012.06242

Gaia Collaboration et al., 2016, A\&A, 595, A2

Gaia Collaboration et al., 2018, A\&A, 616, A1

Gaia Collaboration Brown A. G. A., Vallenari A., Prusti T., de Bruijne J. H. J., Babusiaux C., Biermann M., 2020, arXiv e-prints, p. arXiv:2012.01533

Gao X., 2020a, PASJ, 72, 47

Gao X., 2020b, ApJ, 894, 48

Harris C. R., et al., 2020, Nature, 585, 357

Hu Q., Zhang Y., Esamdin A., Liu J., Zeng X., 2021, arXiv e-prints, p. arXiv:2103.02912

Hunter J. D., 2007, Computing in Science and Engineering, 9, 90

King I. R., 1966, AJ, 71, 64

Lamers H. J. G. L. M., Gieles M., Bastian N., Baumgardt H., Kharchenko N. V., Portegies Zwart S., 2005, A\&A, 441, 117

Mathieu R. D., 1984, ApJ, 284, 643

Mclachlan G., Peel D., 2000, Finite Mixture Models, 44

Meingast S., Alves J., Rottensteiner A., 2021, A\&A, 645, A84

Mermilliod J. C., Mathieu R. D., Latham D. W., Mayor M., 1998, A\&A, 339, 423

Nikiforova V. V., Kulesh M. V., Seleznev A. F., Carraro G., 2020, AJ, 160, 142

Odenkirchen M., et al., 2001, ApJ, 548, L165

Pinfield D. J., Jameson R. F., Hodgkin S. T., 1998, MNRAS, 299, 955

Portegies Zwart S. F., McMillan S. L. W., Gieles M., 2010, ARA\&A, 48, 431

Rao K. K., Vaidya K., Agarwal M., Bhattacharya S., 2021, arXiv e-prints, p. arXiv:2102.07409

Reid M. J., Brunthaler A., 2004, ApJ, 616, 872

Röser S., Schilbach E., 2019, A\&A, 627, A4

Röser S., Schilbach E., Goldman B., 2019, A\&A, 621, L2

Salpeter E. E., 1955, ApJ, 121, 161

Schlafly E. F., Finkbeiner D. P., 2011, ApJ, 737, 103

Spitzer Lyman J., 1958, ApJ, 127, 17

Tang S.-Y., et al., 2019, ApJ, 877, 12

Twarog B. A., Anthony-Twarog B. J., Deliyannis C. P., Thomas D. T., 2015, AJ, 150, 134

Vaidya K., Rao K. K., Agarwal M., Bhattacharya S., 2020, MNRAS, 496, 2402

Virtanen P., et al., 2020, Nature Methods, 17, 261

Wang S., Chen X., 2019, ApJ, 877, 116

Yeh F. C., Carraro G., Montalto M., Seleznev A. F., 2019, AJ, 157, 115

von Hoerner S., 1957, ApJ, 125, 451

This paper has been typeset from a $\mathrm{T}_{\mathrm{E}} \mathrm{X} / \mathrm{L} \mathrm{T} \mathrm{E} \mathrm{X}$ file prepared by the author. 